\title{
Étude sur les tiques du bétail en Guadeloupe et Martinique II. Agents pathogènes transmis par les tiques
}

\author{
par P. C. MOREL
}

\begin{abstract}
RÉSUMÉ
Les protozoaires sanguicoles observés en Guadeloupe chez le bœuf splénectomisé sont Babesıo (Piroplosma) bigernina et Theileria mutans; la piroplasmose bovine vraie apparaît sporadiquement en Guadeloupe et Martinique chez les animaux importés d'orıgine européenne. L'existence de Babesia caballi et de B. canis est probable dans les deux îles, du fait de la présence des vecleurs sur les hôtes sensibles. En ce qui concerne les rickettsiales, Anap'osmo morginale a été vu chez le bœuf splénectomisé et l'anaplasmose clinique est parfois constatée : la présence de Cowdryo ruminantium est vrassemblable en Guadeloupe en relation avec la présence d'Amblyomma voriegatum et du fait de l'analogie des symptômes entre des accès enzootıque aigus observés dans cette île et d'ériologie non encore élucidée avec la symptomatologie de la cowdryose en Afrique : des larves d'Amblyomma variegatum ont été trouvées spontanément infectées par Rickettsı conori. Le bélall de Guadeloupe présente une dermatose analogue ou identique à la streptothricose bovine ofricaine due à Dermatophsius congolensis, et associée comme celte dernière à Amblyomma variegotum.
\end{abstract}

Babesia (Piroplasma) bigemina (Smith ef Kilborne, 1893); Du Toit, 1918.

Le parasite a été mis en évidence à l'occasion de la splénectomı d'un veau d'un an et demi, originaire de la Hatte-Saint-Jacques (GrandeTerre de Guadeloupe) ; ce même animal a fourni les Boophilus utilisés dans les essals insecticides (il étalt d'alleurs très infesté, d'au moins 500 femelles) ; l'état général, plutôt mauvais, accusait surtouł une grande maigreur. La splénectomie a eu lieu le mercredi 29 septembre, avec anesthésie au Nesdonal Spécia ; la rate était très grande ; le lendemain de l'intervention le comportement du veau étalt normal ; il mangeait ef avait retrouvé son agressivité. Les premiers piroplasmes sont apparus le 7 octobre, en même temps que Theilerio mistons ; au début les B. bigemina étaient rares ou peu abondantes (Th. mutons était fréquente) jusqu'au 15-16octobre, après le 18 octobre, les $B$. bigemina sont devenues relativement nombreuses ; Anaplasmo marginale a fait son apparition du 18 au 23 octobre, date de la fin de la mission. Au cours de la parasitémie le veau a encore maigri, par rapport à son état avant la splénectomie : ses múqueuses ont pris une teinte ivoire jaunâtre, sans jamais devenır ictériques; jamais les urines n'ont été colorées. L'animal a été alors tratté au Zothélone Spécia ef rendu à son propriétaire, qui l'avait gracieusement prêté pour les besoıns de l'enquête.

La morphologie de la $B$. bigemina rencontrée en Guadeloupe n'offre aucune particularité par rapport aux descriptions habituelles. Sa présence aux Petites-Antilles est normale, du fait de l'existence de Boophilus microplus, son vecteur naturel sur toute son are de distribution. Une attention spéciale a été portée aux petites formes, le plus souvent trigéminées ; en aucun cas il ne pouvait s'agir de Bobesia (Babe- 
sia) argentina Lignières, 1901) [- Babesıa bovis Starcovicl, 1893 ?]. Cette absence demanderait à être ultérieurement infirmée ou confirmée, car $B$. microplus transmet $B$. argentina concurremment avec B. bigemina en Asie tropicale orientale, en Australıe, à Madagascar et en Amérique néoptropicale.

La piroplasmose bovine à B. (Piroplasma) bigemina existe en Guadeloupe et Martinique. L'affection n'est signalée le plus souvent que sur le bétail amélıoré, au cours de la saison sèche: il en va de même pour les taureaux du pays qui tirent les charrettes de canne, car cette période coincide avec le maxımum de leur travail. En toute saison la maladie présente un très grand danger pour les bovins de race, importés des Etats-Unis ou d'Europe (ce sont le plus souvent des taureaux pour la reproduction). Une piroplasmose grave se déclare dans les $30-45$ jours qui suivent si l'animal n'a pas été survellé et défendu contre les tıques, ou plus tard malgré la surveillance, à l'occasion de la piqûre par quelques Boophilus, ce qui manque rarement de se produire. II s'ensuit des piroplasmoses aiguës ou suraiguës, ordinairement mortelles sans les médications appropriées, compromettant l'état et la productivité du sujet pendant une longue convalescence de plusieurs mois. Partout où existe un élevage amélıré, éclate la piroplasmose.

A titre documentare sont relatés ici quelques cas de cette affection, signalés dans les rapports annuels du Service vétérinaire de Martinique pour ces dernières années ; Il s'agit presque toujours d'animaux importés; en 1955, 5 cas ; en 1956, 6 cas (4 sur bovins Holstem à SaintJoseph, 2 cas à Basse-Ponte sur zébus brahmans); en 1957, 7 cas ; en 1960, 10 cas ; en 1962, 5 cas sur des taurecuux charolais ; en 1963, 7 cas.

Les bovins du pays (taurıns ou zébus) manifestent rarement une piroplasmose clinique et semblent prémunis dès leur première année.

Babesia (Plroplasma) cabal'i (Nuttall et Strickland, 1910); Du Toit, 1918.

La piroplasmose équine est signalée de plusieurs localités de Floride, où son vecteur naturel est Anocentor nitens (ROBY et ANTHONY, 1963,268 ). Elle existe également à Panama, en même temps que $A$. nitens (DARLING, 1913, 197). La maladie est donc possible sur toute l'aire de distribution de cette tique, ef notamment en Guadeloupe ef Martinique. Dans les conditions habituelles la plupart des chevaux antillais se trouvent prémunis dès le jeune âge. II n'en demeure pas moins que la piroplasmose présente un réel danger pour tout cheval importé d'un pays où est inconnue Babesia caballi.

\section{Babesia (Piroplasma) canis (Piana ef Galli-} Valerio, 1895) ; Du Toit 1918.

En ce qui concerne les Antilles, la piroplasmose canine semble n'être signalée que de Puerto Rico (MARTINEZ, 1914, 194). En falt B. canis doit exister chez tous les chiens porteurs de Rhipicephalus sanguineus, qui est son vecteur habituel dans toute la zone pantropicale.

Theileria mutans (Teiler, 1906); Brumpt, 1920.

Dans le sang du veau spénectomisé de Guadeloupe, les Th. mutans ont été souvent abondantes: lors de certanes observations, 50-60 p. 100 des hématies étaient parasitées. La morphologie des diverses formes est conforme aux figurations classiques, avec notamment les aspects en flamme de bougie à chromatıne allongée bacıllıforme. En ce qui concerne les pourcentages des diverses catégories, celui des formes rondes et elliptiques était assez élevé, environ de 60 p. 100 ; ce chiffre se rapproche de celui donné par SERGENT, DONATIEN, PARROT et LESTOQUARD (1945: $566) ; 20+35,6$ p. 100 ; par contre DELPY $(1937,225) n$ 'indique que $6 \div 34$ p. 100.

II ne semble pas exister aux Antılles une pathogénıcité partıculière chez Th. mutans, ce qui est conforme aux données admises généralement pour l'Afrıque et le bassin méditerranéen. Pour des raisons épizootologiques, Il semble que le vecteur de Th. mutans en Guadeloupe soit Amblyomma variegatum, de même qu'en Afrique occidentale et centrale, mais le fait n'est pas expérimentalement démontré. C'est toutefois la seule espèce capable, en accord avec son cycle évolutif et l'ımpossibilité chez les Theileria d'infecter la descendance des tıques par voie transovarienne, de transmettre Th. mutans aussi bien en Afrique occidentale qu'en Guadeloupe.

En fait, le petıt nombre des études sur ce parasite, fréquent mais difficile à utiliser dans les expérimentations en rason de sa pathogénıcité naturelle faible ou nulle, explique le peu de renseignements à son sujet. En Asie orientale, Th. 
mutons [ $=$ Th. orientolis (YAKIMOV et SUDAČENKOV, 1931) ?] est naturellement transmıse par Haemaphysalis bispinosa (et peut-être aussı en Australie). En Afrique australe, Rhipicephalus appendiculatus constitue un vecteur expérımental ; le vecteur ou les vecteurs naturels en Afrique orientale, centrale et occidentale ne sont pas connus; compte tenu de la fréquence de la Theileria, ce sont des tıques communes ou très communes qui sont en couse. En Eurasie méditerranéenne le vecteur naturel de Th. mutans n'est pas découvert; il s'agit peut-être d'une Haemaphysalis ( $H$. punctata, $H$. sulcata?).

Jusqu'à aujourd'hui, Th. mutons n'était mentionnée du contınent américain que par la seule référence de SPLITTER $(1950,134)$, sur des bovins du Kansas. L'abservation de l'espèce en Guadeloupe constitue une deuxième référence, qu'il faut peut-être mettre justement en relation avec la distribution limitée de $A$. variegotum à cette île, à Antigua et à la Martınıque. II serait intéressant à cet égard de rechercher la présence de Th. mutans en Martinique dans la zane restreinte où existe $A$. variegotum, et de comparer les résultats avec la situation dans, tout le reste de l'île, d'où A. variegatum est absent.

Theileria parvo (Theiler, 1904); Betiencourt França et Borges, 1907.

MAUZE et MONTIGNY $(1954,504)$ décrivent une épizootie mortelle sur des bovins importés de Floride et Jamaïque à l'élevage Beauport (Grande-Terre de Guadeloupe) ; le cours de la maladie étalt le plus souvent aıgu ou suraıgu, les symptômes consistant en agitation et convulsions accompagnées de jetage, de salivation et de Jarmolement ; puis apparaissaient des parésies et des chutes; enfin les bêtes mouraient paralysées en 24-48 heures; il n'y avalt ni coloration des urines, ni adénites caractérisées. Lors des examens du sang et des organes a été vue en abondance une Theilerio rapportée à Th. parva; le vecteur Incrımıné était Amblyommo variegatum. Ordinairement ces symptômes sont inconnus sur les bovins du pays ; les bêtes locales seraient donc prémunies contre cette affection indétermınée.

Le diagnostic inquiétont de Th. parva faisait redouter que la terrible thellérıse d'Afrique orientale ne soif établıe aux Antilles : or son existence n'aurait pas pu passer jusqu'à ce jour inaperçue, quand on sait les ravages qu'elle provoque en Afrique. II n'étalt pas possible d'autre part qu'il s'agisse d'une theilériose grave de l'Ancien-Monde puisque ni Rhipicephalus appendiculatus (vecteur de Th. parvo), ni Hyalomma detrifum ou $\mathrm{H}$. excovatum (vecteurs de Th. annulato) ne sont présents aux Antilles (naturellement ou secondairement) ; il ne pourralt s'agır que d'une forme propre au pays. Ultérieurement, Il n'a plus été observé de cas attribuables à une theilériose.

Le plus logique est de considérer que cette sortıe dans les hématıes d'une Theilerio mettait en jeu tout simplement Th. mutons; qui a été trouvée en Guadeloupe au cours de cette mission, et précisément à quelques kılomètres de l'élevage Beauport. La symptomatologie observée a été attribuée aux thielérıoses, ce qui a détourné de rechercher plus loin la véritable cause des accès. Le parasitisme par Th. mutans a pu être fortult ou occasionné par une crise relevant d'une autre étiologie, ce qui a masqué la réalité des phénomènes.

Quel serait-donc l'agent causal de cette pseudothellérlose guadeloupéenne? II faudrait orienter les suspicions en premier lieu vers l'hydrocardite rickettsienne (heart water) due a Cowdryo ruminantium. Le fait que la maladie ait été observée dans une région à $A$. variegatum et qu'elle soit entièrement comparable à la symptomatologie de l'hydracardite telle qu'elle se manifeste en Afrique occidentale et centrale, rend cette supposition des plus vraisemblables. Le reste des considérations à ce sujet sera trouvé dans les pages traitant de Cowdrya ruminantıum.

\section{RICKETTSIALES}

\section{Anaplasmo marginale Theiler, 1910.}

Quelques cas d'anaplasmose bovine sont cités dans les rapports annuels du Service vétérinaire de Martinique, sur du bétail importé : en 1958, 10 cas, dont 2 cas mortels aux environs de Moutte (Fort-de-France) et de Jambette (SaintJoseph) (dıagnostıc sur étalements de sang); en 1959, plusieurs cas, dont à Trois-llets et à Moutte ; en 1962, plusıeurs cas à Saınt-Joseph, Le Vauclın, Saint-Pierre ; en 1963, 10 cas.

Lors de la splénectomie du veau de Guadeloupe, quelques anaplasmes sont apparus du 21 au 23 octobre, c'est-à-dıre 3 semaines après l'opération; ils n'étalent pas très nombreux. 
Malheureusement la fin de la mission a empêché de suivre l'évolution de cette anaplasmose expérimentale.

Les vecteurs naturels d'Anaplosma marginale sont ordinairement les Boophilus sur leur aire commune de distribution; l'existence de B. microplus s'accorde donc avec celle de A. morginale. Par ailleurs les insectes piqueurs jouent un rôle certain dans la transmission mécanique de l'anaplasme (seuls les stomoxes pourraient être en cause aux Petites-Antilles). Enfin A. marginale présente une remarquable aptitude à être transmis, lors d'injections en série sans précautions d'asepsie, par les aıgulles hypodermiques souillées de sang infecté.

\section{Cowdrya ruminantium (Cowdry, 1925) ; Mo- kovskiî, 1947.}

En Afrique, sur toute l'aire de distribution des Amblyomma majeurs du bétail, la cowdryose ou l'hydrocardite rickettsienne (heart water) existe à l'état d'enzootie permanente ; les tiques en cause sont $A$. hebraeum d'Afrique australe, $A$. variegotum d'Afrique orientale, centrale ef occidentale, A. gemma et A. lepidum d'Afrique orientale somalo-masaï. Apparemment tout le cheptel est infecté, en état de prémunité. Les manifestations climatiques apparaissent en certaines occasions de rupture de cet état : perturbations climatıques soudaines, changements de saisons, déplacements importants (changements de zone climatıque ou phytogéographique), passage d'un régime alimentaire à un autre (notamment lors de la mise en stabulation de bêtes auparavant en pâture lıbre). D'autres fois la maladie se déclare en tant que Primo-infection : l'occasion s'en présente chez les jeunes, au cours de la première saison de sortie des tiques dans les mois qui suivent leur naissance, sous les climats de type tropical ou subtropical ; comme cette agression n'est pas la seule que connalssent les jeunes (qui subissent également pour la premıère fois les effets des piroplasmes, anaplasmes, trypanosomes, etc.), conjointement d̀ une alimentation défectueuse qualitativement ou déficitaire, il n'est pas possible de décider de la part effective qui revient à $C$. ruminantium dans la mortalıté à cet âge. Lo maladie se déclare évidemment à tout âge chez les sujets importés de régions indemnes de $C$. ruminontium, avec laquelle Is sont pour la première fois en contact.
Les grandes lignes de la symptomatologie de la cowdryose sont les suivantes, chez les petits. et grands ruminants :

- Forme aiguë :

- montée thermique rapide, jusqu'à 41 $41,5^{\circ} \mathrm{C}$, qui dure autant que la maladie ; à partir du $2^{\mathrm{e}}$ jaur, agitation, tournis, pousse au mur, parfois accès de fureur, ou encore ımmobilisation sur place tête basse, mouvements de succion, grincements de dents, claquements des mâchoires ; puis apparition de clonies et contractures, localisées ou généralisées, d'où torsion de l'encolure, voussure dorsale, déhanchement ; les déplacements sont difficiles, incoordonnés, chancelants, ébrieux, s'accompagnant de fauchage ou de steppage ; les épanchements péricardiques sont plus ou moins fréquents chez le mouton et la chèvre, rares chez le bœuf ; la diarrhée fétide est de règle chez le bouf, rare chez les petıts ruminants; après des chutes plus ou moins nombreuses, l'animal ne se relève plus, manifestant une paraplégie d'importance variabie; se déclarent alors soit des crises convulsives avec galop, pédalage et nystagmus, ou des crises tétaniformes; ordinairement l'animal meurt en hyperthermie, au cours d'une crise ; d'autres fois, ce sera en hypothermie, après une crise; la mort arrive en 2-6 jours; si la guérison est obtenue, la convalescence est longue (plusieurs mois).

- Forme suraigue ;

- forme foudroyante, se déclarant par une chute brusque au sol, montée thermique rapide, jusqu'à $42 \circ \mathrm{oC}$ et accélération respiratare extrême ; l'animal meurt en quelques quarts d'heure ou quelques heures, au cours d'une crise d'agitation convulsive ou d'une crise tétaniforme.

Le tableau clinique dans l'un et l'autre cas est marqué par l'importance des signes nerveux ; Cowdrya ruminantium, localisée dans l'endothélium des vaisseaux, provoque une déformation des cellules et un rétrécissement du diamètre interne des capillaires du cerveau, ce qui entrcîne ischémies et congestions locales, d'où découlent les symptômes de type encéphalitique.

L'existence de Amblyomma variegatum dans certanes îles des Antilles oblıge à y suspecter la présence de la cowdryose. Bien qu'aucune entité pathologique n'y ait jamais été rapportée 
à C. ruminantium, il y a tout lieu de croire que certaines maladies dominées par des signes nerveux et d'étıologie non définie sont à identifier avec la cowdryose ; elles se déclarent dans les régions infestées par $A$. coriegotum. Au cours de la mission en Guadeloupe, II n'a pas été possible d'être témoin de manifestations cliniques de cette sorte. Les symptômes relatés, observés dans divers élevages de races pures bovines, s'apparentent à des crises aiguës ou suraiguës de cowdryose ; jusqu'à présent aucune inferprétation étiologique de ces symptômes n'a été proposée, en l'absence de tout argument d'ordre microbiologique, parasitologique ou sérologique permettant d'orienter un diagnostic.

En 1954, lors des premières apparitions de la maladie à Port-Louis (élevage Beauport, Guadeloupe), la présence concomitante de Theileria nombreuses dans les hématies a condult à un diagnostic de theılérıose à Th. parvo (MAUZE et MONTIGNY, 1954, 504). Cependant aucune adénite n'a été observée (normale dans les theilérioses); de plus l'épizootologie est très différente; dans les theilérioses la morbıdité est élevée, de même que la mortalité, et son existence n'aurait pu passer inaperçue si longtemps en Guadeloupe, même sur du bétail rustique ; enfin Il n'existe dans l'île aucune tique connue comme vectrice de theilériose ( $c f$. Clavant les pages sur Theilerio parva).

Par la suite COURMES et AUDEBAUD (1962, 40), à l'occasion de nouveaux accès mortels dans le même élevage, entre octobre 1960 et février 1961, décrivent les symptômes suivants : hypothermie $\left(39,5-40,5^{\circ} \mathrm{C}\right)$, démarche instable, flexion de la tête, tremblements : puis l'anımal paralysé fombe et meurt en 24-48 heures (parfols en 1-2 heures) ; les lésıons sont discrètes et non caractérıstıques; la monocytose est accusée, avec de grands corps basophiles dans les monocytes (1 à 20), ce qui fat suspecter l'éventualıté d'une rickettsıose des monocytes à Rickettsia bovis [s'agissait-il seulement de grains azurophiles?]. Des réaclions d'agglutinatıon des sérums de malades ont donné dans 3 cas 2 réponses positives à Rickettsia conori ef 1 réponse faible à l'antıgène rickettsien $Q 18$ de Giroud (agent de l'avortement des brebis), sans aucune réponse envers Coxiello burneti; d'autres épreuves sérologiques correspondant à 111 bovins de l'élevage ont montré plusıeurs réponses d'agglutination au 1/80 et au 1/20 à l'antigène de C. burneti. Les traitements à l'auréomycine ont été inefficaces, sauf si la thérapeutique était appliquée précocément. L'épizootie est apparue à l'occasion d'un ralentıssement du rythme des bains antı-tiques et la réapparition de nombreux Amblyomma variegatum en aoûł 1960.

Ici donc l'orientation du diagnostic vers une rickettsıse semble parfaitement judicieuse.

En plus des similıtudes de symptomatologie entre la cowdryose et la maladie de Guadeloupe existent plusieurs autres présomptions :

- La maladie apparaît sur des bovins non prémunis, nouvellement importés ou tenus ordinairement indemnes d'Amblyomma, conditions réalisées dans les élevages importants de type moderne.

- A. variegatum existe en Guadeloupe, où la maladie est signalée, mais non en Martinique, qui ignore cette affection.

- En Afrique moutons et chèvres sont sensibles, même les animaux du pays ; or l'élevage des petits ruminants est difficile en Guadeloupe (Grand-Terre et Basse-Terre) ; ce cheptel est en état d'équilıbre numérique instable; certaines années un grand nombre d'animaux meurt pour des raisons plus ou moins claires (le plus souvent les petits propriétaires ne sıgnalent même pas le fait au Service vétérinaire); par contre des îlots comme la 'Désirade ou les Saintes ont la réputation d'être favorable à l'élevage du mouton ; indépendamment de la sécheresse de la Désırade (mais la plus grande partıe de GrandeTerre lui est comparable de ce point de vue), il se trouve que $A$. variegatum est absent de ces deux îlots ; comme conséquence, 30.000 bovins sont recensés en Martinique, 7.000 seulement en Guadeloupe (et beaucoup sont dans les îlots sus-nommés).

- A plusieurs reprises des prélèvements de sérums de bovins malades ou saıns ont été envoyés dans les services de $M$. le professeur P. GIROUD, d I'Institut Pasteur de Paris ; il y a eu plusieurs réponses positıves aux épreuves d'agglutination avec l'antıgène néorickettsien Q 18 ; sans mettre en doute pour autant l'existence de néorickettsıes en Guadeloupe, $M$. le professeur P. GIROUD signale (communication orale) qu'en ce qui concerne l'Afrique, des 
sérums de bovins, porteurs selon toute vraisemblance de $C$. ruminantium, ont donné des réponses positıves avec ce même antigène $Q 18$.

En conclusion il existe en Guadeloupe une maladie bovine dont la symptomatologie et l'épizootologie sont tout à fait semblables à celles de la cowdryose, ou rickettsiose africaine à Cowdrya ruminantium, concurremment avec la présence d'Amblyomma variegatum. Réciproquement, étant donné l'existence de cette tique en Guadeloupe, l'infection consécutive à C. ruminantrum doit être dès l'abord suspectée.

En fait le diagnostic de cowdryose n'a pas encore été définitivement établi.

En raison de l'ambiguilté des épreuves sérologiques, seule la mise en évidence cytologique de C. ruminantium permettra d'affirmer sa présence. Les éléments parasitaires doivent être recherchés dans le système réticulo-endothélial, soit dans l'endothélium de la veine jugulaire (frottis du produit de râclage de l'endothélium avec un bistouri tenu perpendiculairement), soit dans l'endothélium des capillaires du cerveau, dont l'obstruction provoque les signes encéphalitiques (frottis sur lame par décalque de fragments des zones congestionnées du cerveau).

Des traitements précoces à l'auréomycine confirmeraient la possibilité d'une rickettsiose, s'ils étaient suivis de succès.

\section{Rickeftsia conori Brumpt, 1932.}

Plusieurs cas de typhus rickettsien humain ont été observés en Guadeloupe ces derniers mois, et les épreuves sérologiques ont orienté le diagnostic de l'agent pathogène vers des rickettsies du groupe boutonneux-pourpré. Grâce à l'amitié du Docteur A. ESCUDIÉ, de I'Institut Pasteur de Pointe-à-Pitre, une enquête, tardive il est vrai, a pu être faite sur deux des derniers cos, dans le but de mettre en évidence le vecteur éventuel.

Dans le premier cas, une fièvre boutonneuse s'est déclarée chez un habitant de Bagatelle (LAMENTIN, Basse-Terre) ; ici seuls les commémoratifs peuvent ètre invoqués. Le sujet avait trouvé sur lui, en abondance, au retour d'une journée de chasse dans les environs de SaintFrançois (Grande-Terre), des petites tiques de la taille d'un grain de plomb, une semaine environ avant le début de sa maladie. Au domicile de l'ıntéressé d'autre part vivent des chiens, por- teurs de Rhipicephalus sanguineus. Les tiques rencontrées dans les savanes, à la chasse, sont très vraisemblablement des Amblyomma variegatum ; d'après les résultats de l'enquête sur les tiques des Antilles, c'est la seule espèce et le seul stade capable de piquer régulıèrement l'homme, souvent en grands nombres, dans les conditions ordinaires. Malheureusement l'existence de Rh. sanguineus chez l'ancien malade laisse subsister un doute sur le vecteur possible de la rickettsıse qui a été contractée dans ce cas ; malgré tout, le caractère fortuit du parasitisme par les Amblyomma peut être plus facilement rapproché de l'apparition de la rickettsiose que la présence permanente de $R h$. sanguineus à proximité du malade. En plus des signes cliniques, le diagnostic de fièvre boutonneuse s'appuyait sur une réaction sérologique positive avec l'antigène de Rickettsia conori.

Le deuxième cas concerne un homme de Schoelcher (Vieux-Habitants), dont le domicile est isolé en dehors de l'agglomération, sur un morne couvert de savane bolsée. Il s'agit d'une petite exploitation familiale avec bovins, chèvres, volailles et chiens. Ces derniers sont parasités par Rh. sangumeus; les rumınants par Boophilus microplus et Amblyomma variegatum. L'homme qui a fait la ricketrsıse à la fin de 1964 a pu être en contact à l'accasion avec des larves de A. variegatum abondantes en certaines périodes sur les herbes; il ne peut rien affirmer à ce sujet, n'ayant jamais vu de tique sur lui ; en réalité if ne fait que circuler sur les chemins et petites routes au milieu de la savane pour se rendre à son travail en ville dans une scierie; Il ne s'occupe donc pas en permanence sur sa propriété ; par contre sa femme et sa belle-sœur, chargées des cultures et du petit élevage, sont très affirm atıves : elles observent très souvent des petites tiques sur les herbes et sont fréquemment porteuses de tiques gorgées au retour de leur travail. Lorsqu'll leur a été montré des larves et nymphes gorgées de $A$. variegatum recueillies sur leur bétail, et des larves et nymphes de Rh. sanguineus prises sur leurs chiens, elles ont toutes deux désigné les larves de A. variegatum comme semblables aux tiques trouvées sur elies.

Ainsı exıste dans la région où a été contractée la rickettsiose des $A$. variegatum qui piquent l'homme au stade larvaire; diverses personnes 
qui sont en contact permanent avec la savane sont souvent piquées; elles n'ont pas fait de maladie cliniquement caractérisée ; le sujet qui a fait la maladie est de son côté rarement en rapport avec les tiques ; c'est à ce fait peut-être qu'est dô son typhus clinique.

Une femelle de $A$. variegatum recueillie sur une chèvre de cette famille a pondu et les larves issues ont été confiées à $M$. le professeur $P$. GlROUD ef $M$. le docteur M. CAPPONI, de I'Institut Pasteur de Paris. Or une souche moyennement virulente de Rickettsia conorı en a été isolée sur Meriones shawi, sérologiquement proche de souches provenant de $R h$. sanguineus de chiens de Tunis (souche historique) ou de Dermacentor marginotus d'un bovin de l'Allier (communication orale de $M$. le professeur P. GIROUD).

Cet isolement vient de faire l'objet d'une communication (GIROUD, CAPPONI, ESCUDIÉ et MOREL, Bull. Soc. Poth. exot., 1966, sous presse).

Malgré le caractère limıté de cette trouvaille, le fait peut orienter d'une façon précise les conceptions ef les recherches sur l'épıdémiologie de la fièvre boutonneuse en Afrique centrale et occidentale. Il vient confirmer en effet les résultats d'enquêtes menées en Afrique orientale (HEISCH, MC PHEE et RICKMAN, 1957, 459 ; HEISCH, GRAINGER, HARVEY et LISTER, 1962, 272) : 2 souches de Rıckettsı coroni ont été isolées à partir d'Amblyomma variegatum du Kenya (pris sur bœuf et chien), ainsi qu'à partir d'autres tiques (Haemaphysalis leochi et Rhipicephalus simus) et de cerveaux de rongeurs (Lemniscomys $s p$. ef Otomys sp.). Par alleurs une espèce voisine, A. hebroeum, est reconnue comme réservoir naturel et expérimental de $R$. conori. Enfin divers Amblyomma du continent américaın ont été trouvés spontanément infectés ou ont pu l'être en laboratoire par Rickettsia rickettsi, dont $R$. conori ne représente peut-être qu'une souche de l'Ancien-Monde (A. americanum, A. brassliense, A. cajennense, A. maculatum, A. striatum).

Bien que soit admis le rôle de Rhiprcepholus songuineus dans la conservation de $R$. conori, la part jouée par la tique dans la transmission effective de la rickettsie à l'homme semble réduite tout au monns occasionnelle, discutée en fait, eu égard à la rareté du parasitisme de l'homme par tel ou tel stade de Rh. sanguineus (mass il est vrai que quelques piqûres par des larves risquent de passer Inaperçues). Quoı qu'il en solt, le typhus boutonneux se présente avec une fréquence autrement plus élevée que les piqûres par $R h$. sanguineus, notamment en Afrique tropico-équatoriale, où la tique en question est partout présente dans les habitations. Sans négliger la part qui peut revenir aux acariens trombiculides ou aux puces dans la transmission de diverses autres rickettsioses, il était vraisemblable de supposer que certaines tiques jovent un rôle dans l'épidémiologie des affections du groupe boutonneux-pourpré, dont la présence était décelée par les épreuves sérologiques. Or parmi toutes les espèces de tiques présentes en Afrique occldentale, il ressortait qu'une seule pique l'homme couramment, $A$. voriegatum, et plus précisément au stade larvaire, ce qui passe le plus souvent inaperçu quand les larves sont peu nombreuses (pefite taille, douleur minime ou nulle au point de fixation) ; parfois les nymphes aussi piquent I'homme, mais moins fréquemment. A part cela, bien d'autres espèces sont trouvées sur l'homme non fixées, en train de se déplacer; les cas de fixation véritable sont plus rares. En Afrique occidentale donc, les larves de A. variegotum sont tout particulièrement suspectées de transmettre $R$. conori. La situation est analogue en Guadeloupe. Après la preuve de l'infection spontanée de la tique par la rickettsie, il restera d̀ préciser le rôle effectif dans la transmission naturelle.

\section{Ricketisia (Ehrlichia canis Donatien ef Lesto-} card, 1935.

Cette espèce a été signalée d'Aruba (Antılles méridionales néerlandases) sur le chien (BOOL et SUTMOELLER, 1957, 418).

Il convient de rappeler ici l'hypothèse selon laquelle les rickettsies des monocytes observées chez les carnivores et herbivores domestiques. rattachées au genre ou sous-genre Ehrichia Moshkovskiı, 1937 (E. canis, E. bovis, E. ovina), pourraient être considérées comme des formes adaptatives de $R$. conori, en conséquence de leur situation chez des mammifères évolués réceptifs mais non clinıquement sensibles, entraînant une infection latente avec localisation atypique dans les monocytes (cf. GIROUD, 1964, 6027). Le caractère fragmentaire des connaissances sur leur nature, leur distribution, leurs vecteurs et leur pathogénıcité rendraient compte des diffi- 
cultés d'expérimentation ou d'observation sur un parasite en sifuation anormale, ef ne contredirait pas la supposition formulée plus haut.

\section{AFFECTIONS DIVERSES}

\section{Dermatose bovine (à Dermatophilus congolen- sis Van Saceghem, 1915 ?).}

Une dermatase affectant les bovins plus particulièrement à la reprise de la saison des pluies existe en Guadeloupe. Les lésions touchent les zones déclives (fanon, poitrine, mamelles, région ano-génitale) comme les zones supérieures (épaules, croupe) ; la localisation sur le canon et lepied est fréquente ; les lésions sont indépendantes des points de piqûre des Amblyomma, qui se traduisent par un abcès froid induré cratériforme.

D'après quelques cas observés à Bellevue (Baillif), l'affection semble débuter par une inflammation cutanée; la peau est chaude, rouge, épaisse ; les poils sont dressés. A cette phase préparatoire succède une phase d'état, où les papules recouvrent toute la surface cutanée précédemment érythémateuse ; les papules crèvent et donnent une dermite suintante ; ultérieurement cultivent diverses bactéries sur le tissu cutané mis à vif. A ce stade l'évolution de la maladie reste aiguë ou passe à la chronicité ; les régions lésées se recouvrent de croûtes craquelées qui se détachent en placards, délabrant plus ou morns profondément le derme; dans la forme chronique, après plusieurs semaines les croûtes tombent, laissant le derme sous-jacent net ou présentant des cicatrices tourmentèes; une lésion de falble étendue demeure parfois; les guéris qui rechutent l'année suivante sont condamnés. Lorsque les lésions sont étendues, la résorption de toxines, les pertes en eau et en substances déterminent une forme aiguë qui peut entraîner la mort rapidement, en une semaine. Tout ce tableau clinique correspond soit aux cas effectıvement observés, soit aux descriptions des éleveurs ou des représentants du Service vétérinaire.

Cette symptomatologie concide avec celle de la streptothricose bovine africaine.

Les études cliniques et étiologiques de l'affection ont été surtout menées en Afrique et en Australie. L'agent pathogène en cause est Dermatophilus congolensis (Actınomycétales) ; l'identité de l'agent responsable aux Antilles n'est pas connue. Parmi les nombreuses causes favorisantes invoquées dans l'étiologie de cetfe dermatose, les piqûres par les épıneux peuvent jouer un rôle important ; par ailleurs la participation des Amblyomma variegatum paraît très vraisemblable, sans qu'il soit défini s'il s'agit d'une action directe (inoculation de la bactérie) ou indirecte (intoxination du bovin par la salive des tiques présentes en très grand nombre, surtout en début de saison des pluies, provoquant divers désordres cutanés ; sur ces lésions se développeraient secondairement le Dermotophilus).

Quoi qu'il en soit du point de vue théorique, il a été constaté que les traitements contre les Amblyomma réduisent la fréquence et la gravité de l'affection, ou préviennent son apparition, cela en Afrique orientale et occidentale. Aux Antilies il semble en être de même ; la dermatose existe en Guadeloupe, en même temps que A. variegotum; en Martinique, la maladie n'a été observée que sur l'aire restreinte infestée par cette tique, entre 1950 et 1960 (signalée notamment dans le rapport annuel du Service vétérinalre de Martinique pour 1958). En ce qui concerne les traltements anti-tıques, l'élevage Beauport constitue un exemple précis ; la dermatose bovine, sévissant avant la mise en place des piscines (1953-1954), a disparu depuis i pendant l'été 1963 s'est produite une réapparition momentanée, contemporane d'un retour offensif des Amblyomma pour diverses causes mal éclaircies (voir la publication sur la résistance des tiques aux insecticıdes). II en va de même dans les autres élevages importants de Guadeloupe où le bétail est surveillé et traité, ce qui fournit des arguments de comparason utilisables dans l'étude de la corrélation entre la présence des tiques et celle de la dermatose.

Il faut ajouter qu'une dermatose analogue est signalée d'Antigua, voisine de la Guadeloupe, où existe également $A$. variegatum (SAUNDERS, 1915, 36. FORD, 1919, 45) ; d'après ces textes, la maladie est plus grave les années pluvieuses, sans que le fait solt mis en parallèle avec une recrudescence numérique des Amblyomma.

En conclusion; il existe chez les bovins, dans certaines îles des Antilles infestées par A.variegatum, une dermatose analogue à celle provoquée en Afrique par Dermatophilus congolensis, et très vraisemblablement identique d̀ cette dernière. 


\section{Farcin du bouf à Nocardia farcinica.}

Le farcin du boeuf a été signalé plusieurs fois de Guadeloupe ; sa coincidence avec le parasitisme par les Amblyommo variegotum a fait supposer que cette tique jove un rôle en provoquant une lésion primaire de piqûre, infectée secondairement par l'agent responsable du farcin, à moins que la tique ne soit directement responsable de l'inoculation infectante (cf. XIROUDAKIS, 1935, 112, Hyolomma aegyptium ; SENEVET, 1938, 226). II semble plus vrasemblable que la piqûre de l'Amblyommo inter- vienne au même titre que les autres lésions favorisant l'infection : blessures diverses, atteintes, piqûres par des épineux.

Un texte ancien de COUZIN $(1879,400$ ef 450 , la tique du Sénégal) suppose de même le rôle d'A. variegatum dans la transmission du farcin en Guadeloupe ; en fait l'auteur confond sous ce nom le farcin du bouf (à Nocardio farcinica) et la lymphangite ulcéreuse équine (à Corynebacterium preisz-nocardi), sans qu'il soit possible de décider si le rôle qu'il prête à la tique concerne l'un ou l'autre des agents. pathogènes.

\section{SUMMARY \\ Study on the cattle ticks in Guadeloupe and Martinique. If Pathogenic agents transmitted by ticks}

Following blood protozoa have been found in Guadeloupe in splenectonized cattle : Babesio (Piroplasma) bigemina and theileria mutans the true Bovine Piroplasmosis accurs sporadically in Guadeloupe and Martinique amongst cattle imported from Europe. The presence of ticks on horse and dog suggests the existence of Babesia Cabalii and B. Conis in both Islands. As far as the rickettsiae are concured, Anasplasma marginale was found in splenectonized cattle and disease was sometımes clinacally recorded, in view of the existence of Ambiyomna Variegatum and the fact that some symptoms observed between acute Enzootic outbreaks are similar to those of Cowdrysis in Africa, Cowdryo ruminantium is likely to be prevalent in Guadeloupe : larvae Amblyomno Varlegatum naturally infected by Rickettsia conori have been found.

A Dermatosis similar or identical to the African bovine Streptothricosis caused by Dermatophilus Congolensis occurs amongst Guadeloupean cattle and is related, like this latter one to Amblyomna Variegatum.

\section{RESUMEN}

Encuesta sobre los ixodos del ganada en Guadalupe y Martinica. II. Agenles patogenos transmitidos por las garrapatas

En Guadalupe se encontraron los protozoarios de la sangre siguientes : Babesia (Piroplosma) bigemina y Thelleria mutans en los bovinos esplenectamizados; la verdadera piroplasmosis bovina ocurre esporádicamente en Guadalupe y en Marlinica en los animales importados de origen europea. La presencia de los vectores sobre los huéspedes sensibles sugiere la exisfencio probable de Babesia cabalii y de $B$. conis en las dos islas. En lo que concierne las rickettsiales, Anoplasma marginale estuvo en el bovino esplenectomizado y a veces se comproba la anaplasmosis clinica. La presencia de Amblyommo voriegatum así como las síntomas de los accesos enzooticos agudos, de erialogıa no todavia canocida, semejantes a las de la cowdriosis en Africa indican la presencia verasimil de Cowdrya ruminantium en Guadalupe. Se encontraron larvas de Amblyomma variegotum naturalmente infectadas por Rickeffsia conori. El ganado de Guadalupe presenta una dermatosis análoga o idéntica a la estreptotrıcosis bovina africana provocada por Dermatophilus congolensis y asociada como la última a Amblyomma variegatum. 